\title{
Solar scandium abundance
}

\author{
H. W. Zhang ${ }^{1,2}$, T. Gehren ${ }^{2}$ and G. Zhao ${ }^{3}$ \\ ${ }^{1}$ Department of Astronomy, School of Physics, Peking University, Beijing 100871, P.R. China \\ email: zhw@bac.pku.edu.cn \\ ${ }^{2}$ Institut für Astronomie und Astrophysik der Universität München, Scheinerstrasse 1, \\ D-81679 München, Germany \\ ${ }^{3}$ National Astronomical Observatories, Chinese Academy of Sciences, Beijing 100012,
} P.R. China

\begin{abstract}
We investigate the formation of neutral and singly ionized scandium lines in the solar photospheres. Extensive statistical equilibrium calculations were carried out for a model atom, which comprises 92 terms for Sc I and 79 for Sc II. Synthetic line profiles calculated from the level populations according to the NLTE departure coefficients were compared with the observed solar spectral atlas. Abundance determinations using the ODF model lead to a solar Sc abundance of between $\log \varepsilon_{\odot}=3.07$ and 3.13, depending on the choice of $f$ values.
\end{abstract}

Keywords. Sun: abundances, line: formation, line: profiles

\section{Introduction}

The solar photospheric abundances serve as a reference for abundance determinations in metal-poor stars, so a reliable set of photospheric abundances is important. Ever since Anders \& Grevesse (1989) published their widely used solar elemental abundance table, many revisions and updates to photospheric and meteoritic abundances of the elements have become available, although the solar photospheric scandium abundance has not been updated for quite a long time. The photospheric abundance value of log $\varepsilon_{\odot}(\mathrm{Sc})=3.10 \pm 0.09$ adopted by Grevesse (1984) was changed to $3.05 \pm 0.08$ by Youssef \& Amer (1989). Neuforge (1993) obtained $3.14 \pm 0.12$ from the Sc I lines and $3.20 \pm 0.07$ from Sc II lines. The average value of $3.17 \pm 0.10$ was adopted by Grevesse \& Noels (1993) and was kept in the newest tabular version of Grevesse et al. (2007), which is somewhat higher than the meteoritic value of $3.04 \pm 0.04$.

It should be noted that local thermodynamic equilibrium (LTE) has been assumed in previous papers about scandium abundance determinations, and NLTE investigation of the scandium element has never been published. In general, departures from LTE are commonplace and often quite important, in particular for low surface gravities or metallicities, with minority ions and low-excitation transitions the most vulnerable.

In this paper we investigate the statistical equilibrium and formation of neutral and singly-ionized scandium lines in the solar photosphere.

\section{NLTE line formation calculations}

Our atomic reference model is constructed from 92 and 79 terms for neutral and singlyionized scandium, respectively. The number of bound-bound transitions treated in the NLTE calculations is 1104 for Sc I and 1034 for Sc II. For bound-free radiative transitions in the Sc atom, hydrogen-like photoionization cross-sections are adopted. 
In our calculations for Sc, we take into account inelastic collisions with electrons and hydrogen atoms leading to both excitation and ionization. Because laboratory measurements and detailed quantum mechanical calculations for collision cross-sections are absent, approximate formulae are applied.

\section{Analysis of scandium lines in the solar spectrum}

We investigate the formation of Sc I and Sc II lines in the solar atmosphere and derive the scandium abundance based on spectrum synthesis. Lines in the solar spectrum are calculated using the plane-parallel hydrostatic MAFAGS-ODF solar model atmosphere with $T_{\text {eff }}=5780 \mathrm{~K}, \log g=4.44,[\mathrm{Fe} / \mathrm{H}]=0.00, \xi_{t}=0.90 \mathrm{~km} \mathrm{~s}^{-1}$.

The observed solar flux spectrum was taken from the Kitt Peak Atlas (Kurucz et al. 1984). Spectrum synthesis was employed to determine the abundance of scandium in the solar atmosphere. For the solar abundance analysis we selected 4 Sc I and 17 Sc II lines, which ideally should satisfy the following conditions: they are relatively free from blends, and oscillator strengths and hyperfine splitting parameters are available. Line profiles are computed under both LTE and NLTE assumptions: fitted to the observed profiles by means of scandium abundance variations.

\section{The solar scandium abundance}

Two sets of oscillator strengths are applied and compared in our abundance determinations: (i) theoretical values taken from Kurucz' database (http://kurucz.harvard.edu), and (ii) experimental data of Lawler \& Dakin (1989). Using the values obtained for log $g f \varepsilon_{\odot}$ and the $\log g f$ values from different data sets, we computed Sc abundances for the individual lines.

Under the LTE assumption, absolute solar abundances determined from Sc I lines are significantly lower than the values obtained for the Sc II lines. The mean LTE abundances for $4 \mathrm{Sc} \mathrm{I}$ and $17 \mathrm{Sc}$ II lines are $2.90 \pm 0.09 \mathrm{dex}$ and $3.10 \pm 0.05 \mathrm{dex}$, respectively. This discrepancy of the ionization equilibrium is resolved in NLTE calculations. Under NLTE assumption, Sc I and Sc II lines give very consistent abundance results, i.e. $3.08 \pm 0.05$ dex and $3.07 \pm 0.04$ dex, respectively. Using Kurucz' $g f$ values, the mean abundance for all $21 \mathrm{Sc}$ lines under NLTE is $3.07 \pm 0.04$ dex. Using instead the laboratory $\log g f$ values of Lawler \& Dakin (1989), the mean abundance of 17 Sc lines under NLTE is 3.13 \pm 0.05 dex.

Our abundance result by using Kurucz' $f$-values lead to a solar Sc abundance well in agreement with the meteoritic value, whereas the experimental data of Lawler \& Dakin deviate from that reference by nearly $2 \sigma$.

\section{References}

Anders, E. \& Grevesse, N. 1989, Geochim. Cosmochim. Acta, 53, 197

Grevesse, N. 1984, Phys.Scr., 8, 49

Grevesse, N. \& Noels, A. 1993, in: N. Prantzos, E. Vangioni-Flam, \& M. Casse (eds.), Origin and Evolution of the Elements (Cambridge: Cambridge Univ. Press), p. 15

Grevesse, N., Asplund, M., \& Sauval, A. J. 2007, Space Sci. Rev., 130, 105

Kurucz, R. L., Furenlid, I., Brault, J., et al. 1984, Solar Flux Atlas from 296 to 1300nm, Kitt Peak National Solar Observatory

Lawler, J. E. \& Dakin, J. T. 1989, J. Opt. Soc. Am. B, 6, 1457

Neuforge, C. 1993, in: N. Prantzos, E. Vangioni-Flam, \& M. Casse (eds.), Origin and Evolution of the Elements (Cambridge: Cambridge Univ. Press), p. 63

Youssef, N. H. \& Amer, M. A. 1989, A\&AA, 220, 281 